\title{
ENHANCEMENT OF THE SOLUBILITY AND THE DISSOLUTION RATE OF CANDESARTAN CILEXETIL USING MICROSPONGE TECHNOLOGY
}

\author{
SHAIMAA NAZAR ABD ALHAMMID* \\ Department of Pharmaceutics, College of Pharmacy, University of Baghdad, Iraq. Email: shaimaapharmakon@gmail.com
}

Received: 20 April 2018, Revised and Accepted: 04 June 2018

\begin{abstract}
Objective: The aim of the present study is to enhance the dissolution rate of candesartan cilexetil (CC) using microsponges. Candesartan is therapeutically a potent antihypertensive agent, but it suffers a major drawback of poor oral bioavailability, which is estimated to be $15 \%$ due to its low solubility in the gastrointestinal fluids and hepatic first-pass metabolism.
\end{abstract}

Methods: Eudragit-based microsponges were prepared by quasi-emulsion solvent diffusion method using different drug-polymer ratios (1:1 to 1:6), stirring speeds (250-750 rpm), and emulsifier concentrations (polyvinyl alcohol) (0.05\%-0.083\% w/v). Differential scanning calorimetry (DSC) and Fourier transform infrared spectroscopy (FTIR) study for CC, physical mixtures of drug-polymer, and selected formula were investigated to estimate compatibility of CC with other used ingredients. All formulations were evaluated for particle size, production yield, and loading efficiency. The in vitro drug release study of optimized formulation was performed in phosphate buffer $\mathrm{pH} 6.8$.

Results: The obtained results indicated that formula F3 which contains eudragit RS100 at drug:polymer ratio (6:1) was showed the smallest particle size with higher production yield and loading efficiency. DSC and FTIR study of the physical mixtures of drug and polymer revealed no drug-polymer interaction. The results clearly confirm that the percentage of CC released at 30 min from F3, CC powder, and self-made tablet was 54\%, 20.325, and $38.9 \%$, respectively.

Conclusion: Microsponges technique was considered as a promising system to enhance the solubility and dissolution rate of poor-water soluble CC.

Keywords: Candesartan cilexetil, Eudragit, Microsponges, Polyvinyl alcohol, Quasi-emulsion solvent diffusion method.

(C) 2018 The Authors. Published by Innovare Academic Sciences Pvt Ltd. This is an open access article under the CC BY license (http://creativecommons. org/licenses/by/4. 0/) DOI: http://dx.doi.org/10.22159/ajpcr.2018.v11i9.26816

\section{INTRODUCTION}

In the recent years, there has been a considerable importance given to the development of novel microsponge-based drug delivery systems, in order to modify and control the release behavior of the drugs [1]. Microsponges are polymeric delivery systems composed of porous microspheres. They are tiny sponge-like spherical particles with a large porous surface. Moreover, they may enhance stability, reduce side effects, and modify drug release favorably.

Microsponge technology has many favorable characteristics, which make it a versatile drug delivery vehicle. Microsponge systems are based on microscopic, polymer-based microspheres that can suspend or entrap a wide variety of substances and can then be incorporated into a formulated product such as a gel, cream, liquid, or powder [2]. These particles are capable of carrying both lipophilic and hydrophilic substances and of improving the solubility of poor water-soluble molecules [3].

Candesartan cilexetil (CC) is widely used for the treatment of hypertension and heart failure in clinical application. It is a selective AT1 subtype angiotensin II receptor antagonist. After the oral administration, it undergoes rapid ester hydrolysis to convert to the active Candesartan during absorption in the gastrointestinal tract [4].

CC shows a very poor solubility within the physiological pH range, which could result in incomplete intestinal absorption and a very low systemic exposure.Due to its low solubility and a slow dissolution rate therefore the oral administration of CC has an oral bioavailability of about $15 \%$ which needs to be enhanced. CC is a highly lipophilic compound. Therefore, it is necessary to find a new approach to enhance the oral bioavailability of the drug [5].
In this work, we designed and developed CC microsponges to improve the dissolution rate and enhance the bioavailability. CC microsponges were prepared by quasi-emulsion solvent diffusion method.

\section{MATERIALS AND METHODS}

\section{Materials}

CC was purchased from Shenzhen Nexconn Pharma Techs Ltd, China. Eudragit RS 100, Eudragit RL100, and Eudragit S100 was purchased from Rohm Pharma, Germany. Dichloromethane was obtained from GCC Analytical Reagent, UK. Ethanol was purchased from Sigma-Aldrich, Germany. Methanol was obtained from Thomas Baker chemical, Mumbai, India. Glycerol was obtained from BDH, England. Polyvinyl alcohol (PVA), disodium hydrogen phosphate, and potassium dihydrogen phosphate were obtained from SD Fine Chem limited, Mumbai, India. All other chemicals used were of analytical grades.

\section{Methods}

\section{Preparation of CC microsponges}

The microsponges enclosing CC were fabricated by quasi-emulsion solvent diffusion method. In the present study, ethanol, which is capable of dissolving both the drug and the polymer, was selected as the internal solvent. The inner phase comprises eudragit (as mentioned in Table 1) dissolved in $5 \mathrm{ml}$ of ethanol. Once a clear solution was obtained, then the $\mathrm{CC}$ was added and dissolved through ultrasonication at $35^{\circ} \mathrm{C}$ for 15 min to obtain a homogenous clear solution then $0.5 \mathrm{ml}$ glycerol was added to the mixture.

Followed by drop-wise addition of this mixture into an aqueous solution of PVA (outer phase) with different stirring rates 250, 500, and $750 \mathrm{rpm}$ for $60 \mathrm{~min}$. Subsequently, microsponges were formed due to the ethanol 
removal from the system by evaporation. The prepared microsponges were then filtered, washed with distilled water, and subjected to drying at $40^{\circ} \mathrm{C}$ for $24 \mathrm{~h}$ in hot air oven. Finally, microsponges obtained were weighed to determine the production yield [6]. Various formulation batches were prepared as per Table 1 .

\section{Determination of the production yield}

The percentage of the production yield was calculated from the weight of the dried microsponges (W1) and the sum of the initial dry weight of starting materials (W2) as the following equation:

$\%$ Production Yield $=\mathrm{W} 1 / \mathrm{W} 2 \times 100$

The determinations were done in triplicate [7].

\section{Determination of loading efficiency}

$10 \mathrm{mg}$ of microsponges were accurately weighed. They were powdered and extracted with $100 \mathrm{ml}$ of ethanol. The resulting solution was analyzed for CC content by measuring absorbance in an ultravioletspectrophotometer at $254 \mathrm{~nm}$. The studies were carried out in triplicate. Loading efficiency (\%) was calculated using the following equation [8].

$$
\text { Loading efficiency }=\frac{\text { Actual drug content }}{\text { Theoretical drug content }} \times 100
$$

\section{Particle size measurement}

Determination of the average particle size of CC-loaded microsponges was determined with an optical microscope using a calibrated ocular and stage micrometer under a regular polarized light. A minute quantity of microsponges was spread on a clean glass slide and the average particle size was calculated by measuring 100 particles of each batch [9].

$\operatorname{dav}=\sum \mathrm{nd} / \sum \mathrm{n}$

Where dav is the average diameter of particles $(\mu \mathrm{m}), \mathrm{n}$ is number of particles per group, and $d$ is the middle value $(\mu \mathrm{m})$

\section{Differential scanning calorimetry (DSC)}

Thermal analysis using DSC was carried out on drug, physical mixture of the drug and eudragit RS100, and the selected formula to determine the compatibility between the drug and excipients. Accurately weighed sample was loaded into an aluminum pan and sealed. Samples were run at a heating rate of $10^{\circ} \mathrm{C} / \mathrm{min}$ over a temperature range $0-300^{\circ} \mathrm{C}$ in an atmosphere of nitrogen [10].

\section{Fourier transform infrared spectroscopy (FTIR)}

CC and the optimized formula (F3) were subjected to IR analysis; the samples were scanned in the range from $400 \mathrm{~cm}^{-1}$ to $4000 \mathrm{~cm}^{-1}$ and recorded on an FTIR, using the $\mathrm{KBr}$ disk technique [11].

\section{Scanning electron microscope (SEM)}

The microsponge formulations were visualized by SEM to assess the morphology of the microsponges and surface. Samples were coated with gold-palladium under an argon atmosphere at room temperature, and the morphology of the microsponges was studied with SEM at 5kV [12]

\section{In vitro dissolution study}

The dissolution rates for the prepared microsponges formulae were carried out using USP paddle method at $50 \mathrm{rpm}$ in $900 \mathrm{ml}$ of phosphate buffer H 6.8 containing $0.35 \% \mathrm{w} / \mathrm{v}$ Tween 20 as a dissolution media, maintained at $37 \pm 0.5^{\circ} \mathrm{C}$. The samples were collected at specified time intervals $(5,10,15,20,25$, and $30 \mathrm{~min})$, filtered through Whatmann filter paper and assayed spectrophotometrically at $254 \mathrm{~nm}$. An equal volume of fresh medium, which was pre-warmed at $37^{\circ} \mathrm{C}$, was replaced into the dissolution media after each sampling to maintain a constant volume throughout the test $[13,14]$.

\section{Statistical analysis}

The data obtained from each experiment were subjected to statistical analysis by one-way analysis of variance using Microsoft

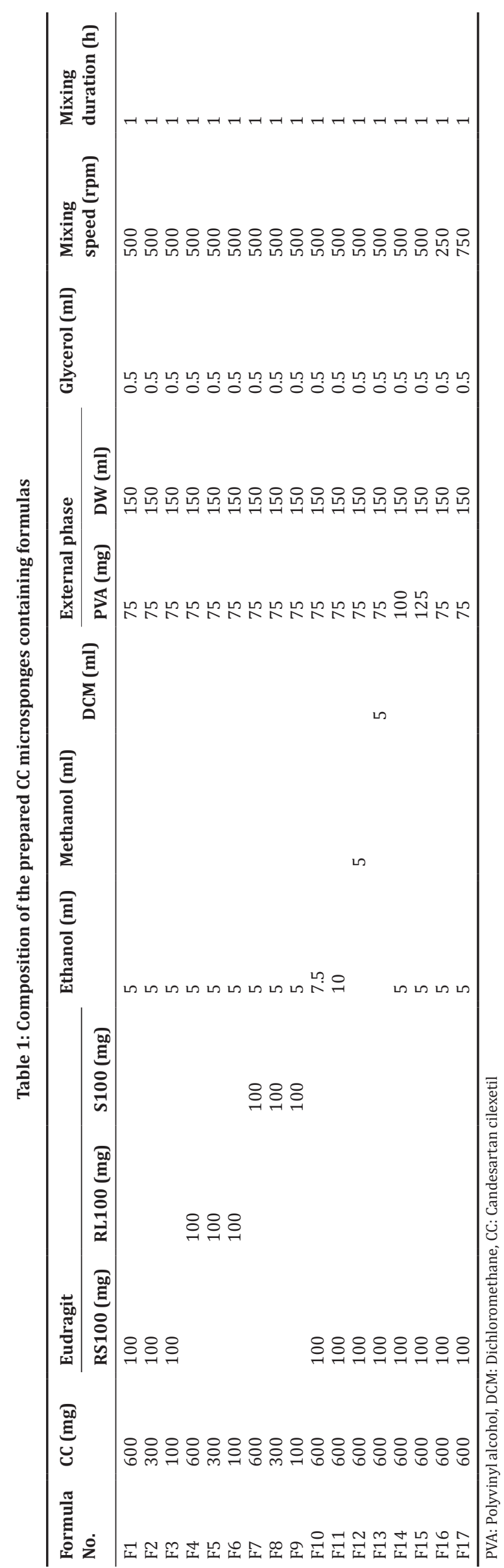


office Excel 2010. $\mathrm{p}<0.05$ was considered to be indicative of significance [15].

\section{RESULTS AND DISCUSSION}

\section{Formulation of CC microsponges}

CC-loaded microsponges were prepared by quasi-emulsion solvent diffusion method which is appeared to be promising for the preparation of CC microsponges as it is easy, reproducible, rapid, and has the advantage of avoiding solvent toxicity [16].

CC-loaded microsponges were prepared from different types of polymer (Eudragit RS100, RL100, and S100) which are biologically inert, non-irritating, non-mutagenic, non-allergenic, non-toxic, and non-biodegradable polymers. As a result, the human body cannot convert them into other substances or break them down, so when these polymers administered orally, they are excreted unchanged with the remaining food [16].

Because of its simplicity and reproducibility, quasi-emulsion solvent diffusion method was used for the preparation of microsponges. Moreover, it has the advantage of avoiding solvent toxicity [10]. The production yield of all formulas lies between 60 and $85.51 \%$, all formulas have an acceptable loading efficiency ranged from 66 to $93 \%$, there is a significant difference $(p<0.05)$ between the three polymer types in case of production yield and loading efficiency. Regarding the particle size of the prepared microsponges, the mean lies between $(79 \mu \mathrm{m}$ and $128 \mu \mathrm{m})$, finally the results are depicted in Table 2.

Effect of formulation variables on loading efficiency, production yield, and particle size

Effect of drug:polymer ratios

First, nine formulas were prepared to study the CC microsponges using different type of polymers (eudragit RS100, RL100, and S100), also different drug:polymer ratio (1:1, 3:1, and 6:1).

The loading efficiencies calculated for all microsponges ranged from $66 \%$ to $89.48 \%$. The highest loading efficiency was found for the F3 formulation which composed of RS100 and higher drug:polymer ratio (6:1), where a greater amount of drug was encapsulated. The highest the loading efficiency, greater the amount of drug was encapsulated. The reason for the increase in EE with high polymer ratios is reduced diffusion rate of drug solution from concentrated polymeric solutions into external phase. As a result, the amount of the drug diffused and flux of the formulations was decreased at a higher drug:polymer ratio. This provides more time for the droplet formation and it may improve microsponges yield and entrapment efficiency [17].

It has been observed that an increase in the drug:polymer ratio resulted in an increase in the production yield as in Table 2. This is because as the drug:polymer ratio went on increasing, the polymer amount available for each microsponge to encapsulate the drug was more, thus rising polymer matrix wall thickness which led to extended diffusion path and ultimately to lesser drug release. As a result, the amount of drug diffused and flux of the formulations was decreased at a higher drug:polymer ratio [18].

The mean particle size of the formulations was between 81.47 and $115.7 \mu \mathrm{m}$. There was a significant difference between formulations $(p<0.05)$ in the mean particle size. The results show that changing drug:polymer ratio has a considerable effect on the size of the prepared CC microsponges. Increasing the polymer fraction significantly increased the particle size $(\mathrm{p}<0.05)$. This could probably be due to increasing the amount of polymer available per microsponge, hence larger particle size was obtained. Previously published data have shown similar findings. Another explanation of this enlargement arouse from the more viscous polymer solution resulting from increasing the polymer fraction. This increase in viscosity hindered the breaking of emulsion into smaller droplets resulting in microsponges with larger particle size [19].

From all the above, microsponges formulae (F3) prepared from RS 100 at the ratio of (6:1) shows higher loading efficiency and production yield $89.48 \pm 1$ and $75.51 \pm 1.5$, respectively. Furthermore, formula F3 showed the smallest particle size $(81.47 \pm 2.1 \mu \mathrm{m})$ therefore will be chosen for studying the effect of other factor on the prepared CC microsponges.

\section{Effect of volume and type of internal phase on microsponges}

Regarding the solvent type, ethanol significantly increases the loading efficiency when compared to methylene chloride and methanol. This may be due to the higher boiling point of ethanol $\left(78.4^{\circ} \mathrm{C}\right)$ compared to methylene chloride $\left(40^{\circ} \mathrm{C}\right)$ and methanol $\left(64.7^{\circ} \mathrm{C}\right)$, so ethanol would evaporate more slowly than methylene chloride. The lower organic solvent evaporation rate led to a lower solvent front kinetic energy, which accordingly decreased the rate of diffusion of the solvent from the inner to the outer phase so increasing the chance for entrapping the drug inside the polymer [20].

However, the particle size was slightly increased (non-significant $\mathrm{p}>0.05$ ) when ethanol is used since a critical parameter determining the particle size seems to be the rate of diffusion of the organic solvent through the interface. At higher diffusion rates, smaller particles are obtained. Increased solvent front kinetic energy causes a higher degree of the droplet dispersion in the aqueous phase [21].

Table 2: Properties of the prepared CC microsponges for the prepared formulas

\begin{tabular}{llll}
\hline Formula & Production yield $\mathbf{~} \mathbf{0} \pm$ SD & Loading efficiency* $\mathbf{0} \pm$ SD & Mean particle size* $(\boldsymbol{\mu m}) \pm$ SD \\
\hline F1 & $67.12 \pm 2.1$ & $72.22 \pm 1.2$ & $101.17 \pm 2.51$ \\
F2 & $76.35 \pm 1.2$ & $70.2 \pm 1.1$ & $95.89 \pm 1.7$ \\
F3 & $85.51 \pm 1.5$ & $89.48 \pm 1$ & $81.47 \pm 2.1$ \\
F4 & $61.52 \pm 1.41$ & $74.87 \pm 1.4$ & $115.7 \pm 2.9$ \\
F5 & $65.74 \pm 1.51$ & $78.8 \pm 2.1$ & $100.57 \pm 1.8$ \\
F6 & $72.47 \pm 1.3$ & $86.23 \pm 1.8$ & $89.4 \pm 1.91$ \\
F7 & $60 \pm 2.7$ & $66 \pm 1.1$ & $106 \pm 1.8$ \\
F8 & $64 \pm 2.4$ & $69 \pm 1.5$ & $95 \pm 2.02$ \\
F9 & $70 \pm 1.7$ & $77 \pm 2.1$ & $87 \pm 2.07$ \\
F10 & $76 \pm 1.2$ & $93 \pm 1.5$ & $79 \pm 1.7$ \\
F11 & & - & - \\
F12 & $83 \pm 1.8$ & $79 \pm 2.1$ & $83 \pm 2.1$ \\
F13 & $78 \pm 1.6$ & $74 \pm 1.4$ & $84 \pm 1.8$ \\
F14 & $75.1 \pm 2.1$ & $77 \pm 1.5$ & $110 \pm 2.1$ \\
F15 & $69 \pm 2.4$ & $69 \pm 2.2$ & $121 \pm 3.2$ \\
F16 & $74 \pm 1.8$ & $76 \pm 1.7$ & $74 \pm 1.5$ \\
F17 & $83 \pm 1.8$ & $84 \pm 1.8$ & $128 \pm 1.2$ \\
\hline
\end{tabular}

*All values are expressed as mean \pm SD, $n=3$. SD: Standard deviation, $C$ : Candesartan cilexetil 
Regarding the volume of ethanol used. It was observed that on increasing the internal phase volume from 7.5 to $10 \mathrm{ml}$ microsponges were not formed. This may be due to the decrease in the viscosity of the internal phase. In this study, it was observed that the particle size, the production yield, and the drug content decreased on increasing the internal phase. These results suggest that the amount of ethanol need to be controlled within an appropriate range to affect not only the formation of quasi-emulsion but also the solidification of the drug and polymer in the droplets [22]. The best microsponges were produced as better when $5 \mathrm{ml}$ of ethanol was used as shown in Table 2 .

\section{Effect of external phase}

The results indicate that changing the external phase concentration $(F 3, F 14$, and F15) caused a significant effect $(p<0.05)$ reduction in the $\mathrm{EE} \%$ and production yield of the prepared CC microsponges as shown in Table 2. This may be because the emulsifier employed (PVA) was nonionic and molecules can associate away from the oil-water interface at higher concentrations. Such alternative hydrophobic region can dissolve some portions of the drug resulting in a reduction in the loading efficiency and the production yield within the microsponge formulations. The results showed that bigger microsponges were obtained when the concentration of PVA was higher. An increase in mean particle size of microsponges with an increase in the emulsifier concentration can be attributed to an increase in the apparent viscosity at increased emulsifier concentrations. Such increased viscosity would result in larger emulsion droplets and finally in greater microsponge size [23,24].

\section{Effect of stirring rate}

It was observed that increasing stirring speed decreases the \% loading efficiency and decrease of the mean particle size. It was found that the stirring rate of the emulsion influences the particle size and its distribution greatly due to the turbulence created within the external phase. It was found that lowering the stirring speed leads to an increase in mean particle due to the increased tendency of globules to aggregation and coalescence, while at higher stirring rates, forceful, even, improved mechanical shear might force and resulted in a rapid dispersion of droplets having less chance of coalescing into bigger droplets [25]

\section{In vitro drug dissolution study}

The dissolution study was performed for the formula that has a better production yield, loading efficiency, and the smaller particle size and also for pure CC powder. The drug release profile of the microsponge formulation showed that cumulative percent drug release was high in the first $1 / 2 \mathrm{~h}$ (Fig. 1).

Tween 20 was selected as a solubilizer due to its ability to increase CC aqueous solubility so that a good sink condition (below $20 \%$ of saturated solubility of drug) could be maintained [14]. The extent of drug release from oral formulations is determined by the dissolution rates of drug, which is a function of aqueous solubility and particle size and it is usually a diffusion-controlled process.

On the other hand, the in vitro drug release reveals that $\mathrm{F} 3$ was found to be better than the self-made tablet and CC powder due to the high thermodynamic activity of drug. The results clearly confirm that the percentage released of CC from F3 reached (54\%) within 30 min, while the CC powder had about (20.325\%) content dissolved after $30 \mathrm{~min}$ and self-made tablet ( $8 \mathrm{mg}$ ) had (38.9\%) content dissolved after $30 \mathrm{~min}$ as shown by Fig. 1 . The porous surface of the carrier particle enables easy penetration of the release media and its accessibility to the entrapped drug molecule.

Burst release was observed, which could be due to the surface adsorbed drug and the well-known porous nature of microsponges, the pores providing channels for drug release. Majority of the drug molecules may reside on the particulate surface as adsorbed molecules that have the probability of undergoing quick solubilization and hence quick drug release. But after achieving equilibrium, the rate of drug release decreased. These results rationalize the importance of the microsponge formulation due to increase of the surface area of the drug which will increase of dissolution rate [16].

\section{SEM study}

SEM of the prepared microsponges revealed a porous nature of the CC microsponges (Fig. 2).

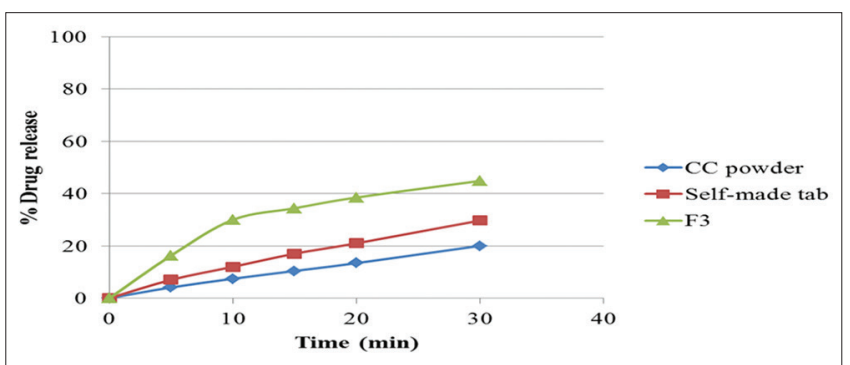

Fig. 1: Cumulative drug release profile of selected formula (F3)

Candesartan cilexetil (CC) microsponges; CC powder; self-made tablet using phosphate buffer $\mathrm{pH} 6.8$ with $0.35 \% \mathrm{w} / \mathrm{v}$ Tween 20 as a dissolution medium, maintained at $37 \pm 0.5^{\circ} \mathrm{C}$
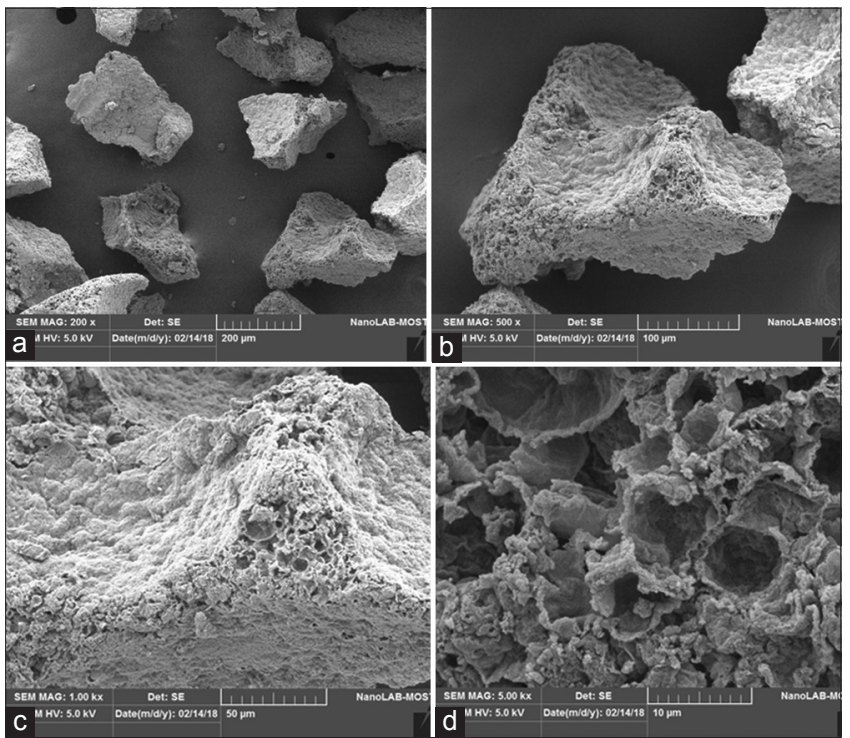

Fig. 2: (a-d) Scanning electron microscope of Candesartan cilexetil microsponges

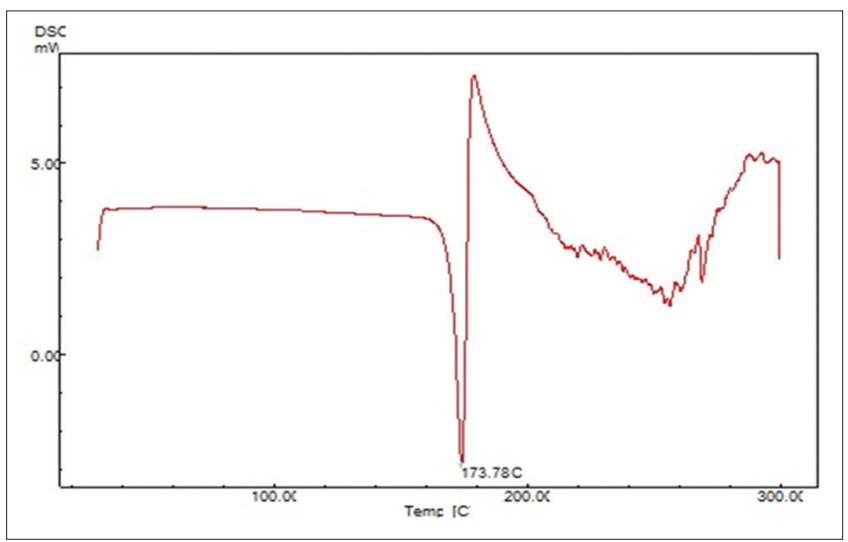

Fig. 3: Differential scanning calorimetry thermogram of a candesartan cilexetil 


\section{Compatibility studies}

\section{DSC study}

The DSC studies are used routinely to evaluate the crystalline state of the drug to demonstrate a possible interaction between the drug and other excipients and to provide information about the physical properties of the drug.

The DSC thermograms of pure CC (Fig. 3) showed a sharp endothermic peak at $175^{\circ} \mathrm{C}$, which is its melting point as it melts with decomposition. Such a sharp endothermic peak indicates that CC used was in a pure

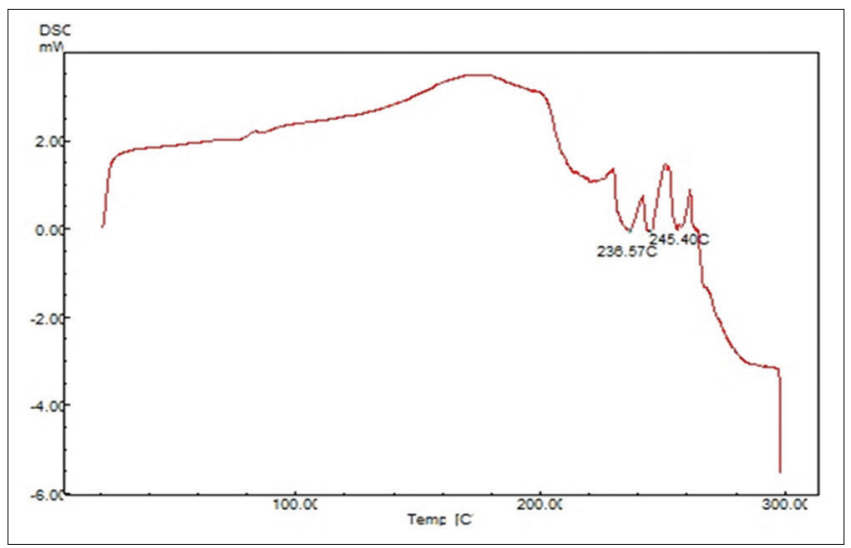

Fig. 4: Differential scanning calorimetry thermogram of candesartan cilexetil microsponges crystalline state [26]. The thermogram of prepared CC microsponges 1:6 (Fig. 4) displayed the microsponges structure is amorphous as no sharp melting peak is shown; this was an expected result because microsponges, as all cross-linked polymers, do not exhibit any melting point [27]

\section{FTIR study}

All the characteristic peaks of CC were present in the spectrum of drug and prepared CC microsponges, indicating compatibility between drug and polymer. The spectrum confirmed that there is no significant change in the chemical integrity of the drug. There is no change in functional group peaks (-C-H, C-N, N-H, C-O-C, C=O) of CC in all the IRspectra and is shown in Fig. 5.

\section{CONCLUSION}

On the basis of obtained results, one can conclude that RS100 was the best polymer for the formulation of CC microsponges. The overall results suggest that drug: polymer ratio of $(6: 1)$ is suitable for the preparation of CC microsponges which shows the higher production yield and loading efficiency and smallest particle size.

\section{ACKNOWLEDGMENT}

The author is very thankful to the College of Pharmacy, University of Baghdad, for providing the necessary facilities to carry out this work.

\section{AUTHOR CONTRIBUTION}

Shaimaa Nazar Abd Alhammid designed the study, developed the methodology, performed the analysis, and wrote the manuscript.

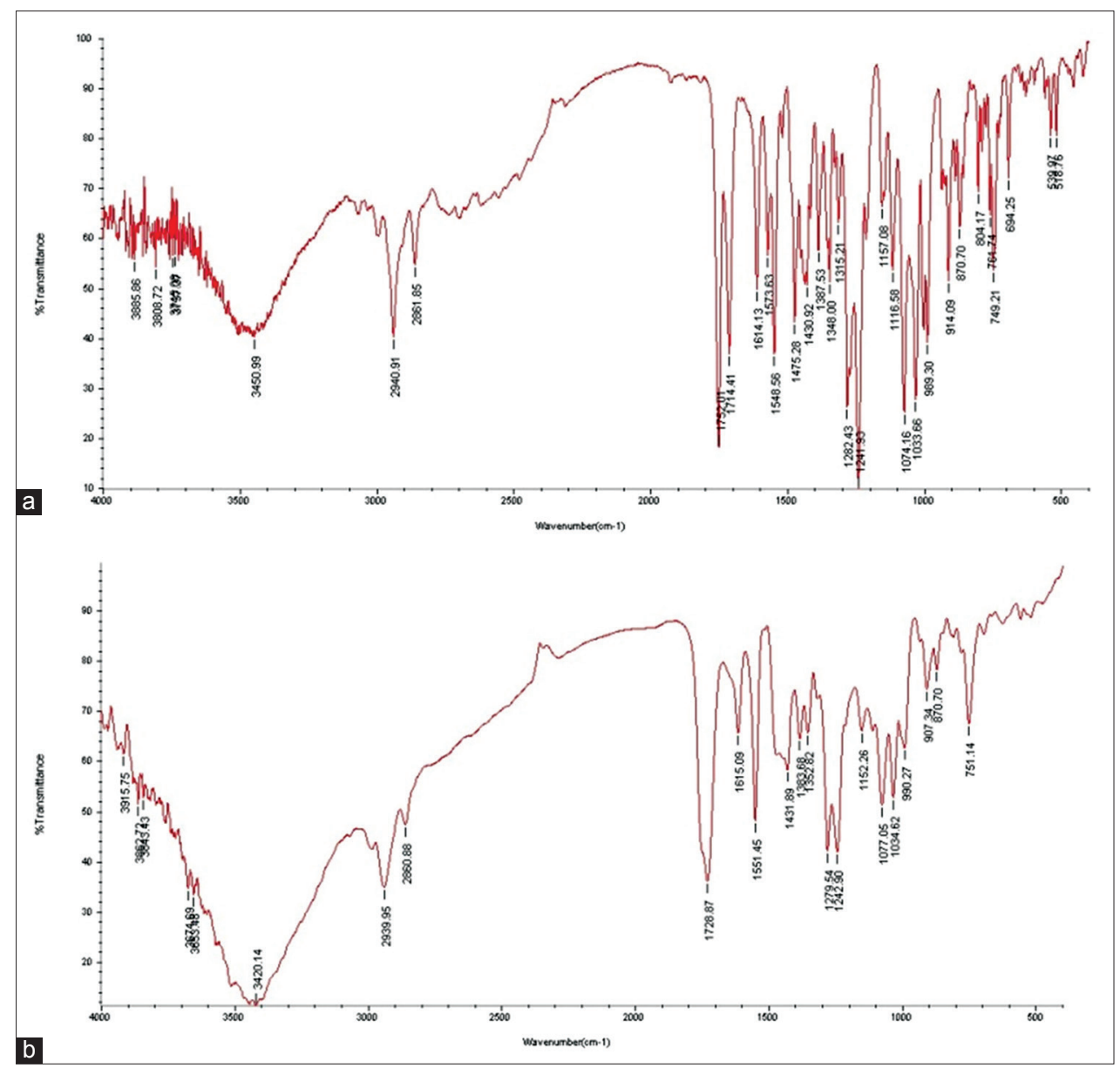

Fig. 5: Fourier transform infrared spectroscopy of (a) pure candesartan cilexetil (CC) (b) CC microsponges 


\section{CONFLICTS OF INTERESTS}

The author declares there is no conflict of interest.

\section{REFERENCES}

1. Ghosh AK, Pal D, Ghosh A, Kaity S. Microsponges: A novel strategy for drug delivery system. J Adv Pharm Technol Res 2010;1:283-90.

2. Charde MS, Ghanawat PB, Welankiwar AS, Kumar J, Chakole RD Microsponge a novel new drug delivery system: A review. Int J Adv Pharm 2013;2:64-70.

3. Thakre AR, Gholse YN, Kasliwal RH. Nanosponges: A novel approach of drug delivery system. J Med Pharm Allied Sci 2016;2:78-92.

4. Salih OS, Nief RA. Effect of natural and synthetic polymers on the properties of candesartan cilexetil matrix tablet prepared by dry granulation. Asian J Pharm Clin Res 2016;9:161-70.

5. Mahajan A, Kaur S. Design, formulation and characterization of stearic acid based solid lipid nanoparticles of candesartan cilexetil to augment its oral bioavailability. Asian J Pharm Clin Res 2018;11:344-50.

6. Desavathu M, Pathuri R, Chunduru M. Design, development and characterization of valsartan microsponges by quasi emulsion technique and the impact of stirring rate on microsponge formation. J Appl Pharm Sci 2017;7:193-8.

7. Sonali S, Singh RP, Prajapati SK. Formulation and evaluation of prednisolone loaded microsponges for colon drug delivery: In-vitro and pharmacokinetic study. Int J Pharm Sci Res 2014;5:1994-2005.

8. Annu P, Anand KY. Design and evaluation of celecoxib microsponge. Int J Pharm Sci Res 2016;7:396-405.

9. Nief RA, Hussein AA. Preparation and evaluation of meloxicam microsponges as transdermal delivery system. Iraqi J Pharm Sci 2014;23:62-74.

10. Jain V, Singh R. Development and characterization of eudragit Rs. 100 loaded microsponges and its colonic delivery using natural polysaccharides. Acta Pol Pharm Drug Res 2010;67:407-15

11. Othman MH, Zayed GM, El-Sokkary GH, Ali UF, Abdellatif AA. Preparation and evaluation of 5-fluorouracil loaded microsponges for treatment of colon cancer. J Cancer Sci Ther 2017;9:307-13.

12. Sareen R, Nath K, Jain N, Dhar KL. Curcumin loaded microsponges for colon targeting in inflammatory bowel disease: Fabrication, optimization, and in vitro and pharmacodynamic evaluation. Bio Med Res Int 2014;2014:340701

13. Prasad BS, Gupta VR, Devanna N, Devi MR, Tamilselvan A. Formulation and evaluation of candesartan cilexetil porous tablets by sublimation technique. J Glob Trends Pharm Sci 2014;5:2125-31.
14. Azim MD, Mitra M, Bhasin PS, Alam MM, Husain A. Development of dissolution medium for candesartan cilexetil by RP-HPLC method. Am J PharmTech Res 2012;2:893-903.

15. Jain V, Singh R. Dicyclomine-loaded eudragit ${ }^{\circledR}$-based microsponge with potential for colonic delivery: Preparation and characterization. Trop J Pharm Res 2010;9:67-72.

16. Rajab NA, Jawad MS. Formulation and in vitro evaluation of piroxicam microsponge as a tablet. Int J Pharm Pharm Sci 2016;8:104-14.

17. Pawar AP, Gholap AP, Kuchekar AB, Bothiraja C, Mali AJ. Formulation and evaluation of optimized oxybenzone microsponge gel for topical delivery. J Drug Deliv 2015;2015:261068.

18. Moin A, Deb TK, Osmani RA, Bhosale RR, Hani U. Fabrication, characterization, and evaluation of microsponge delivery system for facilitated fungal therapy. J Basic Clin Pharm 2016;7:39-48.

19. Abdelmalak NS, El-menshawe SF. A new topical fluconazole microsponge loaded hydrogel: Preparation and characterization. Int J Pharm Pharm Sci 2012;4:460-8.

20. Mainardes RM, Evangelista RC. PLGA nanoparticles containing praziquantel: Effect of formulation variables on size distribution. Int $\mathbf{J}$ Pharm 2005;290:137-44.

21. Orlu M, Cevhe E, Araman A. Design and evaluation of colon specific drug delivery system containing flurbiprofen microsponges. Int $\mathrm{J}$ Pharm 2006;318:103-17

22. Shuhaib B, Suja C. Studies on formulation and characterization of topical gel containing microsponges of mefenamic acid. World J Pharm Life Sci 2018;4:109-19.

23. Nokhodchi A, Jelvehgari M, Siahi MR, Mozafari MR. Factors affecting the morphology of benzoyl peroxide microsponges. Micron 2007;38:834-40.

24. Patel BB, Shah CN. Design and optimization of aceclofenac microsponges containing ethyl cellulose for topical delivery system using quality by design approach. Eur J Biomed Eur J Biomed Pharm Sci 2017;4:495-511

25. Srivastava R, Kumar D, Pathak K. Colonic luminal surface retention of meloxicam microsponges delivered by erosion based colon-targeted matrix tablet. Int J Pharm 2012;427:153-62.

26. Sravya M, Deveswaran R, Bharath S, Basavaraj BV, Madhavan V. Development of orodispersible tablets of candesartan cilexetil- $\beta$ cyclodextrin complex. J Pharm 2013;2013:1-13.

27. Mihailiasaa M, Calderab F. Preparation of functionalized cotton fabrics by means of melatonin loaded $\beta$-cyclodextrin nanosponges. Manuela Carbohydr Polym 2016;142:24-30. 